\title{
Simulación de circuito generador de nanopulsos con herramienta CAD de alto desempeño.
}

\section{Simulation Nano pulse generator circuit with high performance CAD tool}

\author{
Doctorando: \\ Fidel Valentín Giró Uribazo \\ Instituto de Física del Plasma INFIP, Argentina. \\ fgiro841107@gmail.com
}

\section{Director/es:}

\section{Nahuel Olaiz}

\section{Co-director/es:}

\section{Luis Bergues Cabrales}

\section{Resumen}

El efecto de los pulsos de corta duración aún sigue siendo de interés por investigadores como una de las técnicas menos invasivas en diferentes tratamientos médicos, por ejemplo: el cáncer. Los generadores de pulsos Marx son de los más utilizados por ser circuitos muy compactos y de alta escalabilidad modular. Aún persisten muchos problemas en cuanto a acoplamiento y los elementos parásitos que no han sido resuelto ya que no se cuentan con expresiones analítica que permitan con alta precisión determinar la duración y la amplitud del pulso, además de lograr reducir la duración del pulso y aumentar el valor pico del pulso. Todo lo anterior permitió realizar un estudio preliminar de un módulo circuital Marx de 5 etapas con transistores en avalancha(FMMT417) con una herramienta CAD de alto desempeño (ADS) el cual posee varias herramientas de optimización circuital lo cual permitirá mejorar dicho modulo Marx. Como resultado de la primera simulación se logró simular de forma exitosa el generador Marx de 5etapas con una duración del pulso estimado de 1100 V y duración del pulso estimado de 7 ns.

Palabras clave: pulsos, electroporación, electroterapia.

\begin{abstract}
The effect of short duration pulses is still of interest to researchers as one of the least invasive techniques in different medical treatments, for example: cancer. Marx pulse generators are the most widely used because they are very compact circuits and modular scalability. There are still many problems regarding coupling and parasitic elements that have not been solved since there are no analytical expressions that allow determining the duration and amplitude of the pulse with high precision, in addition to reducing the duration of the pulse and increasing the pulse rate pulse peak value. All of the above allowed a preliminary study of a 5-stage Marx circuit module with avalanche transistors (FMMT417) with a high-performance CAD tool (ADS) which has several circuit optimization tools, which will allow to improve said Marx module. As a result of the first simulation, it was possible to successfully simulate the 5-stage Marx generator with an estimated pulse duration of $1100 \mathrm{~V}$ and estimated pulse duration of $7 \mathrm{~ns}$.
\end{abstract}




\section{Introducción}

En el presente las técnicas de electroterapia (ET) como es el caso de la electroporación están brindando excelentes resultados en el tratamiento del cáncer. Dicha terapia emplea electrodos en forma de agujas que se introducen en el tejido de forma directa y se aplica altos campos eléctricos en periodos de tiempo corto permitiendo la ruptura de la membrana celular permitiendo el acceso inmediato de diferentes drogas antitumorales. Aunque es una técnica ya empleada como terapia antitumoral presentan algunos inconvenientes como son: limitaciones en el área a tratar y molestias al paciente por dolores y contracciones musculares (Miklavčič, Nuccitelli, \& Davalos, 2012). Es por ello que se pretende el desarrollo de un dispositivo electroporador sub nsPEF para tratamientos mínimamente invasivos.

\section{Desarrollo}

La ablación con campos eléctricos pulsados de nanosegundos (nsPEF) usa pulsos mucho más cortos (ns) y campos eléctricos más altos $(30 \mathrm{kV} / \mathrm{cm})$. Ambas técnicas se aplican directamente al tumor a través de electrodos de aguja. Los paper más recientes demuestran que los pulsos de duración de picosegundos también pueden causar la muerte celular. Los tratamientos de IRE y nsPEF para tumores sólidos han demostrado en ensayos clínicos (fase I) la reducción del crecimiento tumoral y la inducción de apoptosis de las células tratadas, garantizando seguridad y eficacia (Breton \& Mir, 2011 Aug).

Una de las topologías más empleadas como generadores de subnano pulsos es la topología circuital Marx que ya ha sido estudiada pero que hoy brinda nuevas posibilidades con el empleo de los transistores en configuración de avalancha. Unido a esto las herramientas CAD de alto desempeño brindan la opción de algoritmos de optimización que permitiría optimizar dicha estructura lográndola más compacta y con mejores parámetros de salida.

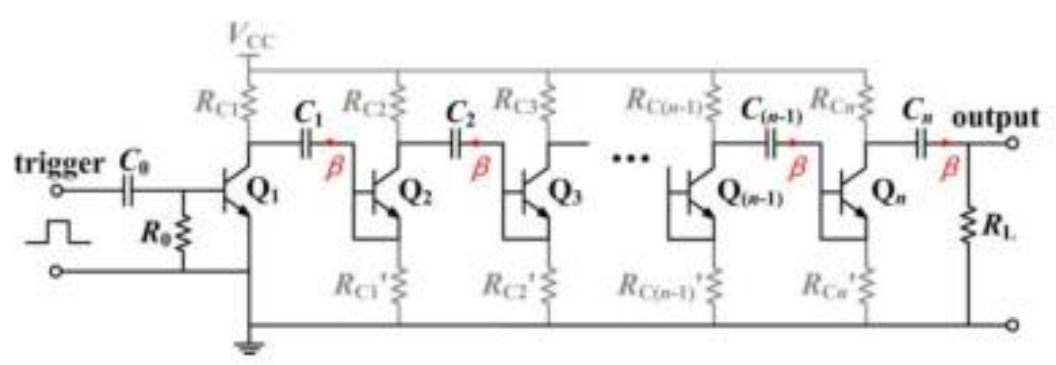

(a)

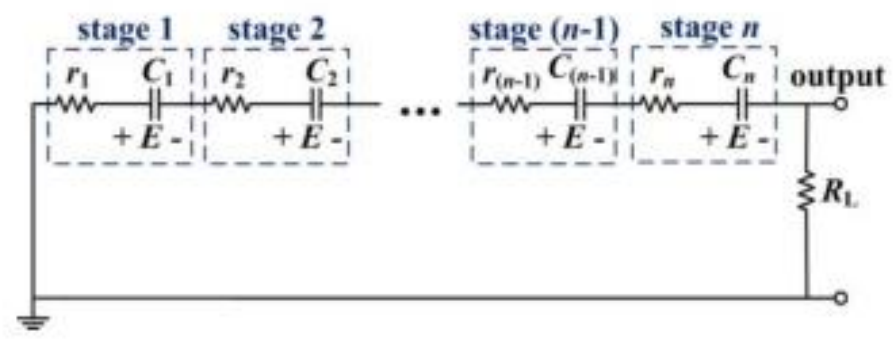

(b)

Fig. 1. (a) Esquemático de un circuito Marx basado en transistores de avalancha. (b) Circuito equivalente de descarga. Las resistencias $R_{i}(\mathrm{i}=1,2, \ldots, \mathrm{n})$ son las resistencias equivalentes de conducción de cada etapa, incluyendo las resistencias de conducción de cada transistor de avalancha el capacitor principal de cada etapa y el efecto de la capacitates parasitas. El voltaje E es el voltaje inicial a través de cada capacitor antes de la descarga.

\section{Calculo y análisis de parámetros del circuito}

Número de etapas Marx: Para estimar el número de etapas Marx , debemos enfocar la atención en el valor pico y se podría considerar despreciable el valor de la resistencia equivalente de los transistores de avalancha. Teóricamente el número de etapas Marx necesario es $V_{m} / E$. Sin embargo, actualmente se requiere un número mayor de etapas debido a los efectos parásitos de los componentes, obteniéndose la siguiente expresión 
Donde:

$$
n=\frac{R_{L} V_{m}}{R_{L} E-r V_{m}}
$$

$n$ : es el número de etapas

E: Voltaje de alimentación

$r$ : Resistencia equivalente

$R_{L}$ : Resistencia de salida (50 $\Omega$ )

$V_{m}$ : Voltaje pico de salida

Es aceptable que la Resistencia equivalente de cada etapa es $r=R_{2-3}+R_{\Delta}$, donde $R_{\Delta}$ es la resistencia equivalente de cada etapa, incluyendo la resistencia equivalente de los capacitores principales así como su capacidad parasita. El efecto de las inductancias despreciable, ya que su efecto es dominante en el tiempo de caída del pulso, pero no en la amplitud del mismo (Heeren, Ueno, \& Wang, 2005). El valor de $r$ se estima entre 2.5 y $3 \Omega$ obtenidos de forma experimental de trabajos anteriores y esto permite obtener un valor de $n=3.06$, para cumplimentar con el valor esperado de $1200 \mathrm{~V}$ de voltaje de salida se escogio 5 etapas.

Capacitores principales: Generalmente los capacitores son seleccionados de igual valor, i.e., $C_{i}=C(i=1,2, \ldots, n)$. Para periodos cortos de descarga, la capacitancia total será $C / n$ como resultado de la ubicación de todos los condensadores en serie [Fig. 1(b)] .El periodo de subida del pulso es determinado por la siguiente expresión de la constante de tiempo ( Ueno, Sakugawa, \& Akiyama, 2009).

$$
\tau_{1}=\left(n r+R_{L}\right) \frac{C}{n}
$$

El pulso de bajada puede ser escrito de la siguiente forma:

$$
\tau_{f}=[\ln (0.9)+\ln (0.1)] \tau_{1} \approx 12.4 C(n s)
$$

Donde:

C: Es la capacidad en $\mathrm{nF}$.

Con un tiempo de bajada estimado $4 \mathrm{~ns}$ aproximadamente se obtiene una $\mathrm{C}$ de $800 \mathrm{pF}$

\section{Resistencia de carga:}

Cada vez luego de la descarga la Fuente de DC recarga todos los capacitores principales a través de la resistencia de carga y el tiempo de carga es proporcional a otra constante $\tau_{2}=R_{2} C$ detreminando esta constante de tiempo la frecuencia de trabajo de repetición del pulso $f$ donde se ha fijado $R_{C i}=R_{C i}^{\prime}=R_{C} / 2(i=1,2, \ldots, n)$. Para garantizar la carga completa de los capacitores en el intervalo del pulso, el periodo de trabajo no debe ser mayor de $5 \tau_{2}$, i.e., $1 / f \geq$ $5 R_{C} C$ (Jiangtao, Zhong, \& Jianhao, 2015).

Donde:

$$
R_{C} \leq \frac{1}{5 f_{c}}
$$

Con una $\mathrm{f}=10 \mathrm{kHz}$ y C $=800 \mathrm{pF}$, se obtiene $R_{C} \leq 25 \mathrm{k}$. Para $R_{C i}^{\prime}={ }_{C} / 2$ se escoge $R_{C i}^{\prime}=10 \mathrm{k} \Omega$

Uno de los objetivos principales de la investigación era modelar mediante una herramienta CAD un módulo circuito Marx de 5 etapas el cual se tenían los resultados con herramientas de bajo desempeño que no permitía la optimización del circuito. Para ello se seleccionó el ADS (Advanced Design Suite) para la simulación a partir del diseño obtenido en la literatura con el objetivo de corroborar dicho circuito, elemento básico de la investigación. Para la simulación se empleó el transistor FMMT417 el cual se tuvo que incluir en las bibliotecas del software, además de modificar la descripción del transistor adaptándolo a los bloques elementales que posee el ADS. 
A continuación, se muestra una imagen con la captura de pantalla del esquemático del circuito a modelar (Fig 2) y la simulación donde se observa una amplitud aproximada de $1100 \mathrm{~V}$ y una duración del pulso aproximada de $7 \mathrm{~ns}$

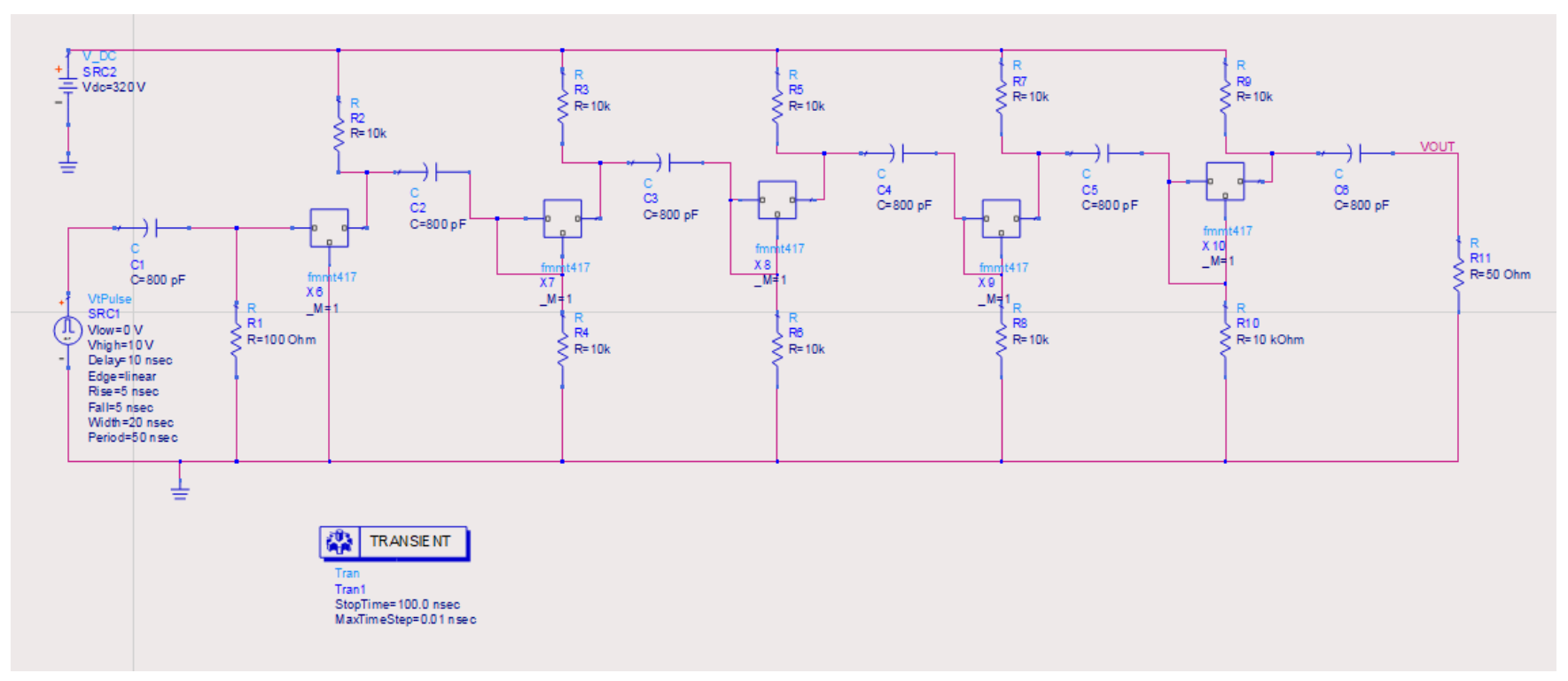

Fig. 2. Esquemático de un circuito Marx de 5 etapas basado en transistores de avalancha FMMT417 a simular en el ADS.

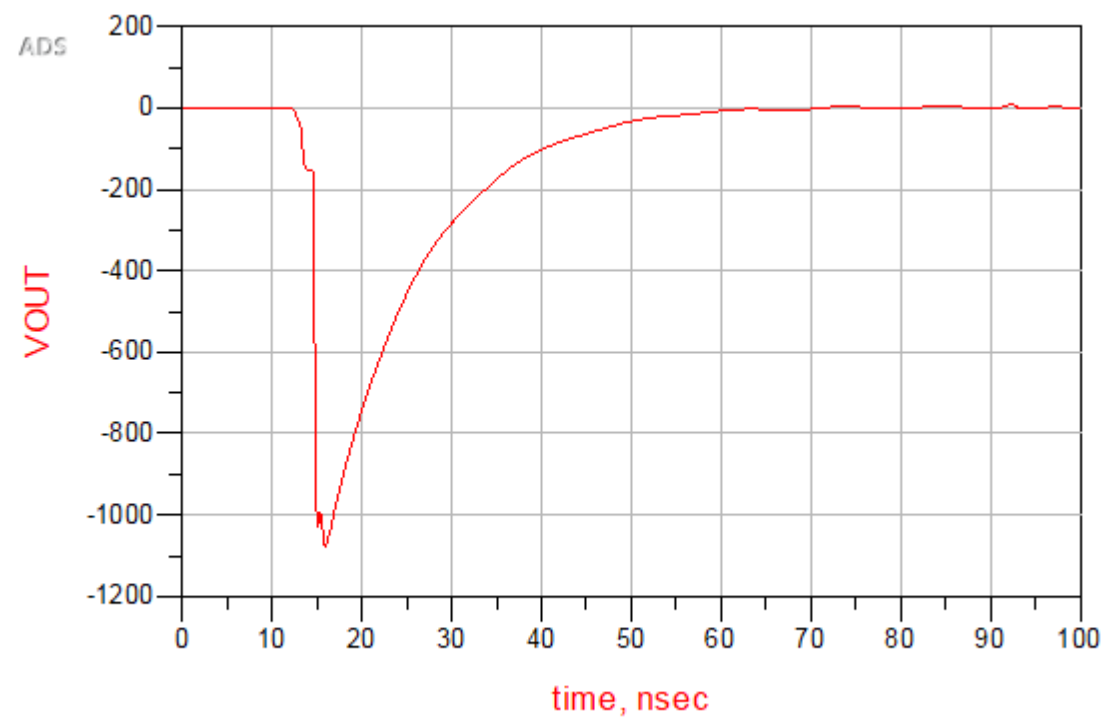

Fig. 3. Simulación temporal con la herramienta ADS del circuito Marx de 5 etapas. 


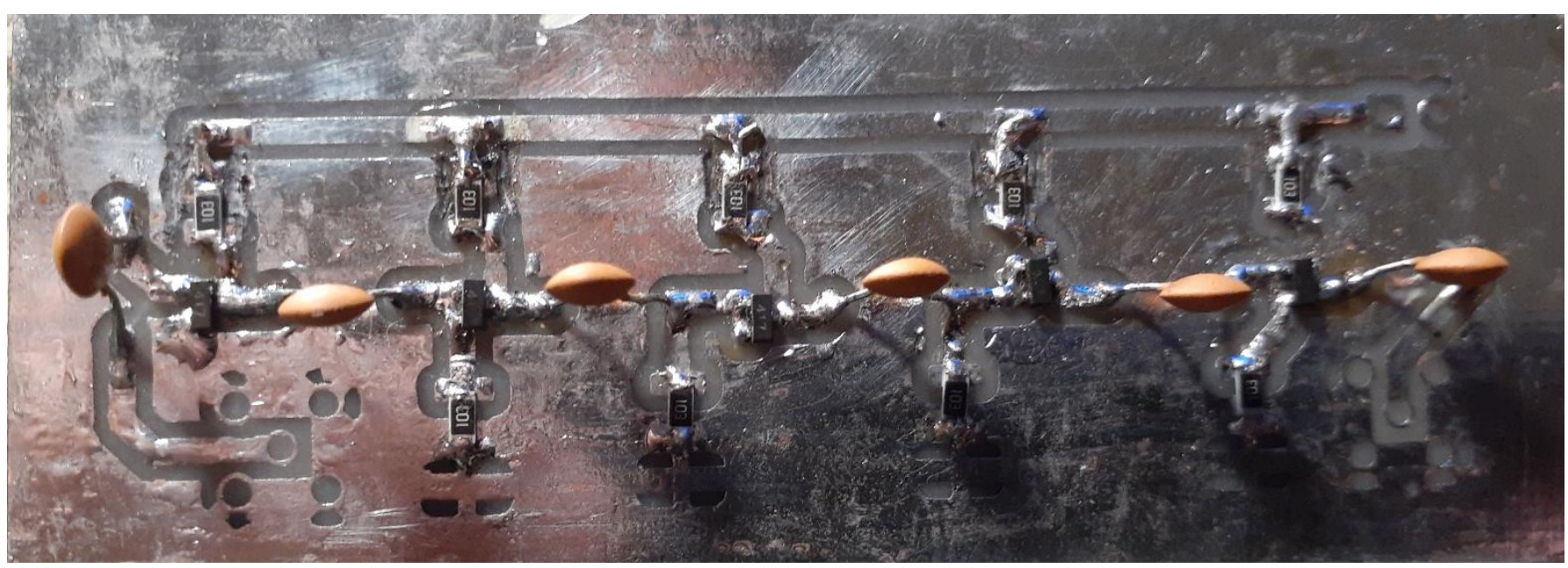

Fig. 4. PCB del circuito Marx de 5 etapas.

\section{Resultados}

Se estudiaron diferentes topologías de generadores de pulsos de corta duración y se escogió la topología Marx con transistores de avalancha en nuestro caso el FMMT417 por su diseño compacto y alta estabilidad de la estructura mediante la optimización circuital de los elementos pasivos inter-etapas.

Se simulo y comprobó el funcionamiento del circuito obteniendo valor esperados de duración $\left(V_{m}>1000 \mathrm{~V}\right)$ y amplitud del pulso $(\sim 7 n s)$, aunque se pretende mejorar dichos parámetros optimizando la estructura.

Con el empleo de la herramienta CAD ADS se pretende además mejorar el acoplamiento de dichas etapas que ocasiona la disminución de la amplitud del pulso, elemento indispensable para la correcta electroporación de las células que se estudiaran en un modelo vegetal.

\section{Referencias}

Ueno, T., Sakugawa, T., \& Akiyama, M. ( vol. 8 de Sep de 2009). Portable Marx generator for microplasma applications. J. Plasma Fusion Res. Ser, págs. 1339-1343.

Breton, M., \& Mir, L. (2011 Aug). Microsecond and nanosecond electric pulses in cancer treatment. Bioelectromagnetics, doi: 10.1002/bem.20692.

Heeren, T., Ueno, T., \& Wang, D. (Vol 33 no4 de Aug de 2005). Novel dual Marx generator for microplasma applications. IEEE Trans. Plasma Sci, págs. 1205-1209.

Jiangtao, L., Zhong, X., \& Jianhao, L. ( OCTOBER de 2015). Theoretical Analysis and Experimental Study on an Avalanche Transistor-Based Marx Generator. IEEE TRANSACTIONS ON PLASMA SCIENCE, págs. VOL. 43, NO. 10.

Miklavčič, D., Nuccitelli, R., \& Davalos, R. (2012). Pulsed Electric Fields in Cancer Treatment. Eslovenia: Cabuy E. 